\title{
Eliashberg theory of excitonic insulating transition in graphene
}

\author{
Jing-Rong Wang ${ }^{1}$ and Guo-Zhu Liu ${ }^{1,2}$ \\ ${ }^{1}$ Department of Modern Physics, University of Science and Technology of China, Hefei, Anhui 230026, P. R. China \\ ${ }^{2}$ Institut für Theoretische Physik, Freie Universität Berlin, Arnimallee 14, D-14195 Berlin, Germany
}

\begin{abstract}
A sufficiently strong Coulomb interaction may open an excitonic fermion gap and thus drive a semimetal-insulator transition in graphene. In this paper, we study the Eliashberg theory of excitonic transition by coupling the fermion gap equation self-consistently to the equation of vacuum polarization function. Including the fermion gap into polarization function increases the effective strength of Coulomb interaction because it reduces the screening effects due to the collective particlehole excitations. Although this procedure does not change the critical point, it leads to a significant enhancement of the dynamical fermion gap in the excitonic insulating phase. The validity of the Eliashberg theory is justified by showing that the vertex corrections are suppressed at large $N$ limit.
\end{abstract}

PACS numbers: 73.43.Nq, 71.10.Hf, 71.30.+h

The low-energy elementary excitations of graphene are known to be massless Dirac fermions with linear dispersion [1, 2]. When Dirac fermions are strictly massless, the system has a semimetal ground state and its Hamiltonian possesses a continuous chiral symmetry. However, when the Coulomb interaction is sufficiently strong, the massless fermion (particle) may combine with an anti-fermion (hole) to form a stable excitonic pair [3, 4]. As a consequence, the Dirac fermion acquires a finite mass gap, which dynamically breaks the chiral symmetry, and the system undergoes a semimetal-insulator transition. In recent years, this excitonic transition has been investigated by several tools, including Dyson-Schwinger gap equation [3 7], Monte Carlo simulation [8 11], and renormalization group [12]. In most of these research works, it was found that such transition can take place when Coulomb interaction strength parameter $\lambda$ is larger than certain critical value $\lambda_{c}$. The opening of fermion gap can result in important changes in the low-energy properties [4, $13-16]$.

Currently, there is still no compelling experimental evidence for the existence of excitonic insulating transition in graphene. In general, there are three possible reasons why the excitonic transition has not yet been unambiguously observed in experiments. First, the Coulomb interaction may be too weak to induce excitonic pair formation. Second, the Coulomb interaction is sufficiently strong in certain graphene materials, but the excitonic transition is suppressed by various fluctuations and perturbations. Actually, we have recently examined this possibility and showed [5, 6 ] that even for sufficiently strong Coulomb interaction the excitonic transition can be destroyed by thermal fluctuations, finite doping, disorder scattering, and finite lattice effect. Third, the excitonic transition does happen in some graphene, but the fermion gap is too small in magnitude to be clearly resolved by any experimental instruments. At present, it is not possible to judge what exactly the reason is. In order to examine the third possibility, it is necessary to determine the magnitude of fermion gap precisely.

Most previous research efforts focused on an accurate determination of the critical point $\lambda_{c}[3-11,16]$. However, an accurate determination of dynamical fermion gap should be equally important. As discussed above, the predicted excitonic insulator is experimentally detectable only when the fermion gap is sufficiently large. On the other hand, the graphene with a large gap will have many technological advantages [16]. As emphasized by Castro Neto [16], if the fermion gap is too small, the interests of excitonic insulating transition would be purely academic. Therefore, an exact determination of the dynamical fermion gap is very important both experimentally and technologically.

In most previous gap equation analysis of the excitonic transition, the vacuum polarization function appearing in the Coulomb interaction function was calculated using the free propagator of massless Dirac fermion, which amounts to assuming the random phase approximation (RPA). Thus the feedback effect of the dynamical fermion gap on the Coulomb interaction was simply ignored. The importance of such feedback effect can be seen by making a simple qualitative analysis. As shown in previous works [5, [6], the screening of Coulomb interaction due to the collective particle-hole excitations can have very important influence on the excitonic transition because it reduces the effective interaction strength. Technically, the screening of Coulomb interaction is described by the polarization function $\Pi(\mathbf{q})$. At the level of RPA, $\Pi(\mathbf{q}) \propto|\mathbf{q}|$, so the Coulomb interaction is weakened due to dynamical screening. Once a finite fermion gap $m$ is included in the polarization function, we have $\Pi(\mathbf{q}) \propto \mathbf{q}^{2} / m$. This term is much smaller than the term $\propto|\mathbf{q}|$ in the low momentum regime, so the effective Coulomb interaction becomes much stronger. From this qualitative analysis, we know that the feedback effect of the dynamical fermion gap may play an important role in the gap equation analysis of the excitonic transition.

In order to compute the dynamical fermion gap more accurately, it is crucial to study the equations of fermion gap and polarization function in a self-consistent manner. This formalism corresponds to the Eliashberg theory [17], which was originally developed to describe the unusual properties of conventional superconductors with strong electron-phonon coupling [17]. Apart from its remarkable success in studying electron-phonon interaction induced 
superconductors [17], the Eliashberg theory is also useful in many other condensed matter problems. In particular, it is widely adopted when studying the non-Fermi liquid behaviors in some correlated electron systems with singular fermion-boson interactions 18 24].

In this paper, we study the Eliashberg theory of the excitonic gap generation in graphene. To make a general analysis, we include both finite temperature and finite chemical potential. After solving the self-consistent equations of fermion gap function and polarization function, we found that the critical point does not change, which is reasonable because bifurcation theory ensures that the fermion gap can be safely taken to be zero near the critical point. However, the size of dynamical fermion gap is significantly enhanced compared to that obtained using the RPA polarization function. This implies that the self-consistent Eliashberg theory plays a crucial role in an accurate computation of dynamical fermion gap. The Eliashberg theory is justified only when the vertex corrections are unimportant. Within the $1 / N$ expansion, we show that the vertex corrections are suppressed in the large $N$ limit.

The Hamiltonian for interacting Dirac fermions is

$$
\begin{aligned}
H= & v_{F} \sum_{i=1}^{N} \int_{\mathbf{r}} \bar{\psi}_{i}(\mathbf{r}) i \gamma \cdot \nabla \psi_{i}(\mathbf{r}) \\
& +\frac{1}{4 \pi} \sum_{i, j}^{N} \int_{\mathbf{r}, \mathbf{r}^{\prime}} \bar{\psi}_{i}(\mathbf{r}) \gamma_{0} \psi_{i}(\mathbf{r}) \frac{e^{2}}{\left|\mathbf{r}-\mathbf{r}^{\prime}\right|} \bar{\psi}_{j}\left(\mathbf{r}^{\prime}\right) \gamma_{0} \psi_{j}\left(\mathbf{r}^{\prime}\right) .
\end{aligned}
$$

As usual, we adopt the four-component spinor field $\psi$ to describe Dirac fermion and define the conjugate spinor field as $\bar{\psi}=\psi^{\dagger} \gamma_{0}$. The $4 \times 4 \gamma$-matrices satisfy the Clifford algebra [3, 4]. The physical flavor of Dirac fermion is $N=2$. The total Hamiltonian possesses a continuous chiral symmetry $\psi \rightarrow e^{i \theta \gamma_{5}} \psi$, where $\gamma_{5}$ anticommutes with $\gamma_{\mu}$, which will be dynamically broken once a nonzero fermion gap is generated.

The free propagator for massless Dirac fermion is $G_{0}^{-1}\left(p_{0}, \mathbf{p}\right)=\gamma_{0} p_{0}-v_{F} \gamma \cdot \mathbf{p}$. After including the interaction effect, it is modified to

$$
G^{-1}\left(p_{0}, \mathbf{p}\right)=A_{0} p_{0} \gamma_{0}-v_{F} A_{1} \gamma \cdot \mathbf{p}-m\left(p_{0}, \mathbf{p}\right)
$$

where $A_{0,1}\left(p_{0}, \mathbf{p}\right)$ is the wave function renormalization and $m\left(p_{0}, \mathbf{p}\right)$ is the fermion gap function. These quantities can in principle be obtained by solving the following complete Dyson-Schwinger equation

$$
G^{-1}(p)=G_{0}^{-1}(p)+\int \frac{d^{3} k}{(2 \pi)^{3}} \gamma_{0} G(k) \Gamma_{0} V(p-k)
$$

where $\Gamma_{0}$ is the vertex function and $V(q)$ is the Coulomb interaction function. The bare Coulomb interaction is $V_{0}(q)=\frac{e^{2}}{2 \epsilon_{0} v_{F}|\mathbf{q}|}$, which is clearly long-ranged. After taking into account dynamical screening effect from collective particle-hole excitations, the effective Coulomb interaction function is modified to

$$
V(q)=\frac{1}{V_{0}^{-1}(q)+\Pi(q)}
$$

with $\Pi(q)$ being the vacuum polarization function. The above gap equation has been studied extensively in recent years and dynamical gap generation was found when Coulomb interaction is sufficiently strong [3-7]. In most of these treatments, only the leading order of $1 / N$ expansion was kept. In particular, both wave function renormalzation and vertex corrections were neglected [3-7], so that $A_{0,1}=1$ and $\Gamma_{0}=\gamma_{0}$. Moreover, the massless fermion propagator is widely used when calculating the polarization function shown in Fig.1 and the feedback effect of fermion mass is simply ignored. Within this approximation, the polarization function has the form

$$
\Pi(q)=\frac{N}{8} \frac{\mathbf{q}^{2}}{\sqrt{q_{0}^{2}+v_{F}^{2}|\mathbf{q}|^{2}}} .
$$

The aim of this work is to go beyond the popular RPA calculation and include the dynamical fermion gap back into the polarization function shown in Fig.1. The importance of this treatment can be readily seen by making a simple qualitative analysis. Generally, the effective strength of Coulomb interaction is characterized by two ingredients: interaction parameter $\lambda=e^{2} N / 16 v_{F} \epsilon_{0}$ with $\epsilon_{0}$ being the dielectric constant and static/dynamical screening due to collective particle-hole excitations. While the former is determined by the substrate of graphene sample, the latter is reflected in the polarization function. Within RPA, the polarization function (5) behaves as $\propto \mathbf{q}$ in the static limit $q_{0}=0$. It vanishes linearly as $\mathbf{q} \rightarrow 0$, so the Coulomb interaction remains long-ranged. However, the effective Coulomb interaction is weakened by the dynamical screening term $\propto \mathbf{q}$, which arises from particle-hole excitations. In 


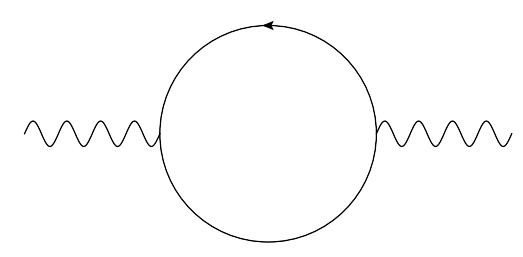

FIG. 1: The bubble Feynman diagram for the polarization function. The solid line is the free fermion propagator and the wavy line is the Coulomb interaction function.

the chiral symmetry broken phase, the Dirac fermion has a finite mass. Assuming a constant mass $m$, the polarization function becomes

$$
\Pi(q)=\frac{N}{\pi} \mathbf{q}^{2}\left(\frac{m}{2 q^{2}}+\frac{q^{2}-4 m^{2}}{4 q^{3}} \arcsin \frac{q}{\sqrt{q^{2}+4 m^{2}}}\right) .
$$

In the limit $q \ll m$, it is simplified to

$$
\Pi(\mathbf{q}) \propto \frac{\mathbf{q}^{2}}{m} .
$$

At the low energy regime, this contribution is much less than the term $\propto \mathbf{q}$ of the polarization function obtained using the massless fermion propagator (RPA). Physically, this reflects the fact that a finite fermion gap reduces the dynamical screening effect. Because of this reduction, the effective Coulomb interaction becomes much stronger.

In general, the fermion mass gap is not a constant, but depends explicitly on momentum. To examine the feedback effect of fermion gap on excitonic transition, we will utilize the Eliashberg formalism and couple the fermion gap equation to the equation of polarization function self-consistently.

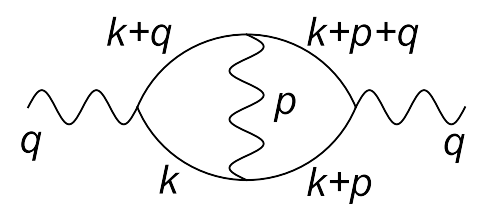

FIG. 2: The polarization operator with the vertex correction, but without self-energy correction to intermediate fermions.

In the Eliashberg formalism, the vertex corrections are usually ignored. This approximation is well-justified in the electron-phonon interacting systems as the Migdal theorem ensures that the vertex corrections are suppressed by a small factor $m / M$, where $m$ is the electron mass and $M$ is the nuclei mass [17]. In the present problem, we still have a small suppressing factor in the vortex corrections based on the $1 / N$ expansion. This can be explained by considering the vertex correction diagram for polarization shown in Fig.2. In the large $N$ limit, it is found (details are presented in the Appendix) to have the form

$$
\Pi_{\mathrm{v}}(q)=-\frac{8}{\pi^{2}} \frac{\ln \left(N \frac{e^{2}}{8 \epsilon_{0} v_{F}}\right) \ln \left(\frac{\Lambda}{q}\right)}{N} \Pi(q) .
$$

Apparently, we know that

$$
\frac{\Pi_{\mathrm{V}}(q)}{\Pi(q)} \propto \frac{\ln \left(N \frac{e^{2}}{8 \epsilon_{0} v_{F}}\right)}{N},
$$

so the polarization function with vertex correction $\Pi_{\mathrm{v}}(q)$ is suppressed by a small factor $\ln (N) / N$ comparing with the leading polarization function $\Pi(q)$, and therefore can be neglected in the large $N$ limit. One can verify in a similar way that the same is also true with the vertex correction diagram for the fermion self-energy. Similar arguments for the suppression of vertex corrections are extensively used in the Eliashberg theories of a plenty of physical problems, 
including fermion-gauge systems [18, 19], quantum critical points in itinerant electron systems [20 23], and electrondoped cuprate superconductors [24]. Moreover, the wave function renormalization $A_{0,1}\left(p_{0}, \mathbf{p}\right)$ also contain certain powers of $1 / N$. Therefore, in this paper we will ignore both vertex corrections and wave function renormalizations.

In order to make a more general analysis, we consider the problem at finite temperature $T$ and finite chemical potential $\mu$ and study how the static screening of Coulomb interaction due to finite $T$ and $\mu$ is affected by fermion mass. We will work in the Matsubara formalism and replace the fermion energy $p_{0}$ by an imaginary frequency, $p_{0} \rightarrow i \omega_{n}=i(2 n+1) \pi T$. At finite $T$, it is convenient to adopt an instantaneous approximation [3, 4] and ignore the energy-dependence of the interaction function. At finite $\mu$, the frequency $i \omega_{n}$ appearing in fermion propagator should be replaced by $i \omega_{n}-\mu$. The instantaneous approximation allows us to perform the frequency summation over $k_{0}$ analytically, leading to the gap equation

$$
\begin{aligned}
m(\mathbf{p})= & \frac{1}{4 N} \sum_{\alpha= \pm 1} \int \frac{d^{2} \mathbf{k}}{(2 \pi)^{2}} \frac{m(\mathbf{k})}{\sqrt{\mathbf{k}^{2}+m^{2}(\mathbf{k})}} \\
& \times \tanh \left(\frac{\sqrt{\mathbf{k}^{2}+m^{2}(\mathbf{k})}+\alpha \mu}{2 T}\right) \frac{1}{\frac{|\mathbf{q}|}{8 \lambda}+\frac{1}{N} \Pi(\mathbf{q}, T)},
\end{aligned}
$$

where $\mathbf{q}=\mathbf{p}-\mathbf{k}$. We need to compute the polarization function $\Pi(\mathbf{q}, T)$ that contains the dynamical fermion mass function $m(\mathbf{q})$. Within the instantaneous approximation, the polarization function is defined as

$$
\Pi(\mathbf{q})=-\frac{N}{\beta} \sum_{n} \int \frac{d^{2} \mathbf{k}}{(2 \pi)^{2}} \operatorname{Tr}\left[G\left(\omega_{n}, \mathbf{k}\right) \gamma_{0} G\left(\omega_{n}, \mathbf{k}+\mathbf{q}\right) \gamma_{0}\right]
$$

where the Dirac fermion propagator $G\left(\omega_{n}, \mathbf{k}\right)$ contains dynamical fermion mass $m(\mathbf{p})$. Following the procedure presented in a previous work [5], we can sum over imaginary frequency $\omega_{n}$ and then obtain

$$
\begin{aligned}
\Pi(\mathbf{q}, T)= & \frac{N}{2} \sum_{\alpha= \pm 1} \int_{0}^{1} d x \int \frac{d^{2} \mathbf{k}}{(2 \pi)^{2}} \frac{1}{t^{2}} \\
& \times\left[\frac{t^{\prime}}{t} \tanh \left(\frac{t+\alpha \mu}{2 T}\right)+\frac{t^{\prime \prime}}{2 T} \frac{1}{\cosh ^{2}\left(\frac{t+\alpha \mu}{2 T}\right)}\right],
\end{aligned}
$$

where we defined three parameters

$$
\begin{aligned}
t= & {\left[\mathbf{k}^{2}+x^{2} \mathbf{q}^{2}+2 x \mathbf{k} \cdot \mathbf{q}+(1-x) m^{2}(\mathbf{k})\right.} \\
& \left.+x m^{2}(\mathbf{k}+\mathbf{q})+x(1-x) \mathbf{q}^{2}\right]^{1 / 2}, \\
t^{\prime}= & x \mathbf{q}^{2}+(2 x-1) \mathbf{k} \cdot \mathbf{q}+(1-x) m^{2}(\mathbf{k}) \\
& +x m^{2}(\mathbf{k}+\mathbf{q})-m(\mathbf{k}) m(\mathbf{k}+\mathbf{q}), \\
t^{\prime \prime}= & 2 \mathbf{k}^{2}+x \mathbf{q}^{2}+(2 x+1) \mathbf{k} \cdot \mathbf{q}+(1-x) m^{2}(\mathbf{k}) \\
& +x m^{2}(\mathbf{k}+\mathbf{q})+m(\mathbf{k}) m(\mathbf{k}+\mathbf{q}) .
\end{aligned}
$$

Now we obtain the self-consistent equations (10) and (11) for dynamical fermion mass function and polarization function. The dynamical fermion mass can be obtained by solving them numerically.

Here, we would like to make some remarks on the instantaneous approximation. This approximation was originally proposed in the study of dynamical fermion gap generation at finite temperature in $\mathrm{QED}_{3}[25$, , 26]. Technically, when calculating the fermion gap equation and polarization function, it is possible to sum over the imaginary frequencies in the Matsubara formalism only when the energy (frequency) dependence of polarization function is neglected. Otherwise, there will be infinitely many coupled gap equations [25, 26]. Moreover, in order to make a qualitative analysis of the screening effects, we need to obtain an (semi)analytical expression for the polarization function, as Eq.(11), which can be derived only within the instantaneous approximation. At zero temperature and zero chemical potential, it is formally viable to include the energy dependence of the polarization function. Indeed, we have solved the fermion gap equation without assuming the instantaneous approximation [5], but using the RPA polarization function (5). The effects of the dynamical part of the RPA polarization function was also investigated in a recent paper of Gamayun et al. 7]. Due to the non-relativistic nature of the present Coulomb-interacting system, the integrations over energy and momenta have to be performed separately, which substantially increases the time needed to perform numerical computations. In the Eliashberg formalism, we need to go beyond the RPA level and solve the self-consistently coupled equations for the fermion gap and the polarization function. This requires much more 


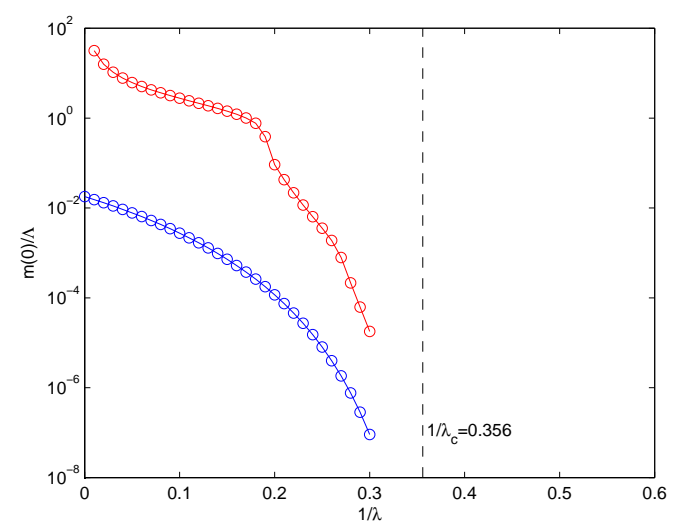

FIG. 3: The $\lambda$ dependence of dynamical fermion gap at zero momentum and zero temperature. At physical flavor $N=2$, the critical strength is given by $1 / \lambda_{c}=0.356$. When $\lambda<\lambda_{c}$, the excitonic insulating transition can not happen.

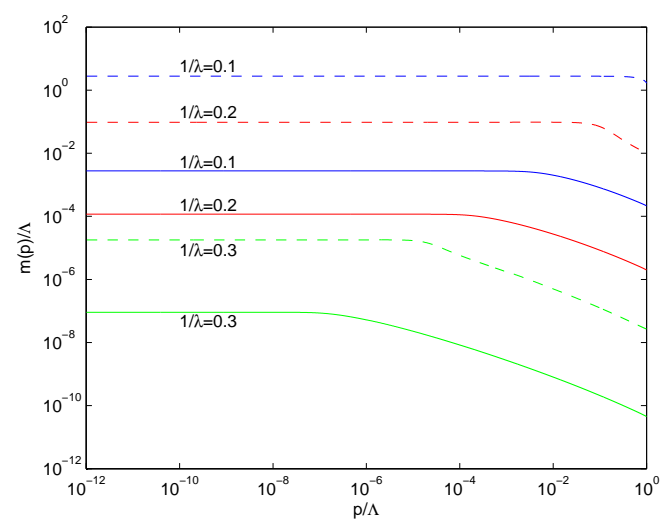

FIG. 4: Dynamical fermion gap at zero temperature.

computer time and would significantly reduce the precision of numerical output. In order to retain the necessary numerical precision, we use the instantaneous approximation even at zero temperature and zero chemical potential.

Before performing numerical computation, it is helpful to first qualitatively analyze the effect of finite fermion gap on the static screening of Coulomb interaction. As an example, we consider the case of finite chemical potential at zero temperature. At $T=0$, the fermion gap equation is simplified to

$$
m(\mathbf{p})=\frac{1}{N} \int \frac{d^{2} \mathbf{k}}{8 \pi^{2}} \frac{m(\mathbf{k})}{\sqrt{\mathbf{k}^{2}+m^{2}(\mathbf{k})}} \frac{\theta\left(\sqrt{\mathbf{k}^{2}+m^{2}(\mathbf{k})}-\mu\right)}{\frac{|\mathbf{p}-\mathbf{k}|}{8 \lambda}+\frac{1}{N} \Pi(\mathbf{p}-\mathbf{k})},
$$

which couples to the polarization function

$$
\Pi(\mathbf{q})=N \int_{0}^{1} d x \int \frac{d^{2} \mathbf{k}}{(2 \pi)^{2}}\left[\frac{t^{\prime}}{t^{3}} \theta(t-\mu)+\frac{t^{\prime \prime}}{t^{2}} \delta(t-\mu)\right] .
$$

Now we assume a constant fermion mass gap $m$, then the integration over momentum $\mathbf{k}$ in these equations can be carried out exactly. When $\mu>\sqrt{m^{2}+\mathbf{q}^{2} / 4}$, the polarization function behaves as $\Pi(\mathbf{q})=N \mu / \pi$. This expression implies that the Coulomb interaction is now statically screened by finite chemical potential and thus becomes shortranged. Such static screening effect will rapidly destroy the excitonic pairing instability [5]. However, when the fermion gap is relatively large, $m>\mu$, we have $\Pi(\mathbf{q}) \propto \mathbf{q}^{2} / m$ in the low momentum regime, so the Coulomb interaction remains long-ranged and is only poorly screened. From this qualitative analysis, we know that once the feedback effect of fermion gap is included, the static screening may be suppressed and the effective Coulomb interaction may still be strong even at finite chemical potential. Besides chemical potential, thermal fluctuation can also induce static screening [5]. The effect of fermion gap on static screening at finite temperature can be qualitatively analyzed 
similarly. To gain more quantitative understanding on feedback effect of fermion gap, we have to perform numerical computation.

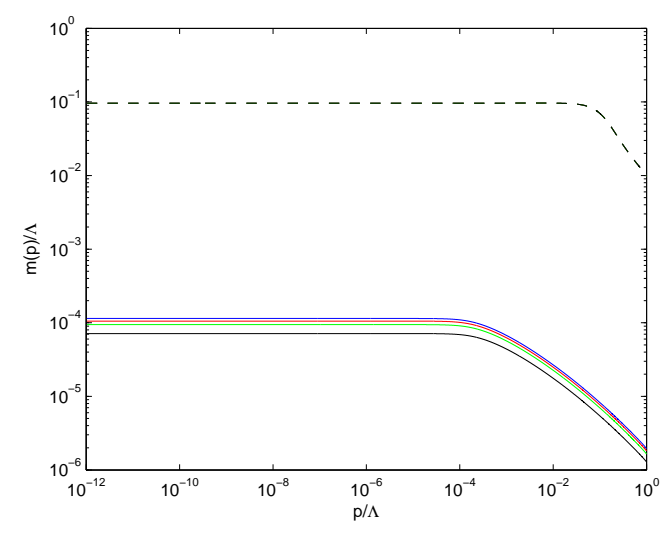

FIG. 5: Dynamical fermion gap at finite temperature and zero chemical potential, with $1 / \lambda=0.2$.

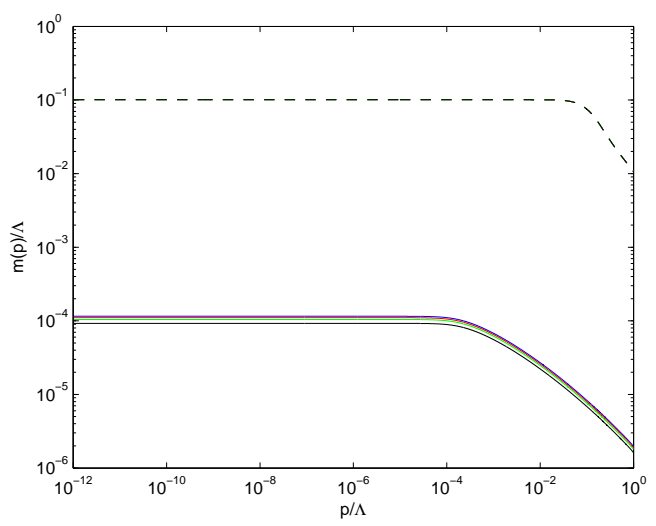

FIG. 6: Dynamical fermion gap at finite chemical potential with $T / \Lambda=10^{-7}$ and $1 / \lambda=0.2$.

We have numerically solved the coupled equations of dynamical fermion gap and polarization function by means of straightforward iterative method. When $T=0$, the fermion gap of zero momentum is shown in Fig 3 , where the blue (red) curve represents the results obtained using the perturbative (self-consistent) polarization function. From the numerical results, we know that the critical Coulomb interaction parameter $\lambda_{c}$ takes the same value in these two cases $\left(1 / \lambda_{c}=0.356\right.$ for physical flavor $\left.N=2\right)$. This fact is easy to understand since the bifurcation theory requires that the fermion gap should vanish near the critical point between gapless and gapped phases. However, away from the critical point in the insulating phase, the fermion gap obtained using different polarization functions are no longer the same. The momentum dependence of fermion gap at $T=0$ is shown in Fig 4 for different values of $\lambda$. The solid (dashed) lines are the results obtained using perturbative (self-consistent) polarization function. The blue, red, and green lines correspond to $1 / \lambda=0.1,0.2,0.3$ respectively. From both Fig. 3 and Fig 4 it is easy to see that the dynamical fermion gap is significantly enhanced in the Eliashberg formalism. Specifically, for graphene suspended in the vacuum with $1 / \lambda=0.295[16]$, the fermion gap calculated by the Eliashberg theory is greater by two orders of magnitude than that calculated using perturbative polarization.

It is not hard to include the effects of temperature and chemical potential. The dynamical fermion gap at finite $T$ and zero $\mu$ is shown in Fig [5, and the fermion gap at finite $\mu$ with $T / \Lambda=10^{-7}$ is shown in Fig 6 . In both of these diagrams, the solid (dashed) lines are the results obtained using pertubative (self-consistent) polarization function. The interaction strength is fixed at $1 / \lambda=0.2$. The blue, red, green, and black lines are the results at $T / \Lambda=10^{-6}, 5 \times 10^{-6}, 10^{-5}, 2 \times 10^{-5}$ respectively in Fig 5 and are the results at $\mu / \Lambda=10^{-6}, 5 \times 10^{-6}, 10^{-5}, 2 \times 10^{-5}$ respectively in Fig 6 Here, $\Lambda$ is the ultraviolet cutoff for momenta. Apparently, there are substantial enhancement effects of dynamical fermion gap in both cases of finite temperature and finite chemical potential. In the Eliashberg 
theory, the static screening effects caused by finite temperature and finite chemical potential are suppressed, so the fermion gap is nearly independent of $T$ (see Fig 5 ) and $\mu$ (see Fig 6 ).

The fermion gap enhancement can be understood as follows. It is well-known that the screening of Coulomb interaction is determined by the density of states of fermions. Once the feedback of dynamical fermion gap is taken into account, the density of states of fermions is significantly reduced and the static or dynamical screening effect becomes less important. As a consequence, the effective Coulomb interaction becomes stronger, which in turn leads to larger fermion gap.

In summary, we have studied the Eliashberg theory of excitonic phase transition in graphene. After solving the coupled equations for dynamical fermion gap and polarization function in a self-consistent manner, we found a significant enhancement of dynamical fermion gap in the excitonic insulating phase. The enhancement found within Eliashberg formalism is owing to the suppression of static or dynamical screening of Coulomb interaction by dynamical fermion gap. Therefore, the self-consistent treatment of polarization function should be used in the accurate computation of the dynamically generated fermion gap. The validity of the Eliashberg theory is justified by showing that the vertex corrections are suppressed by a small factor in the large $N$ limit.

This work is supported by the National Natural Science Foundation of China under grant No.11074234. G.Z.L. is also supported by the Project Sponsored by the Overseas Academic Training Funds of University of Science and Technology of China.

\section{Appendix}

The polarization function $\Pi_{\mathrm{v}}(q)$ shown in Fig.2 is defined as

$$
\begin{aligned}
\Pi_{\mathrm{v}}(q)= & -N \int \frac{d^{3} k}{(2 \pi)^{3}} \int \frac{d^{3} p}{(2 \pi)^{3}} \operatorname{Tr}\left[\gamma_{0} G_{0}(k+q) \gamma_{0} G_{0}(k+p+q) \gamma_{0}\right. \\
& \left.\times G_{0}(k+p) \gamma_{0} D(p) G_{0}(k)\right] .
\end{aligned}
$$

To calculate this function, we will follow the method of Franz et al. 27]. We are mainly interested in the leading behavior of $\Pi_{\mathrm{v}}(q)$ in the $q \rightarrow 0$ limit. In this limit, the above integral has singularities as $k \rightarrow 0$ and $k \rightarrow-p$. Thus, we may evaluate the whole integral by expanding the regular parts of the integrand near these two singular points. Keeping only the leading terms, we have

$$
\begin{aligned}
\Pi_{\mathrm{v}}(q)= & -N \int \frac{d^{3} k}{(2 \pi)^{3}} \int \frac{d^{3} p}{(2 \pi)^{3}} \operatorname{Tr}\left[\gamma_{0} G_{0}(k+q) \gamma_{0} G_{0}(p+q) \gamma_{0}\right. \\
& \left.\times G_{0}(p) \gamma_{0} D(p) G_{0}(k)\right] \\
& -N \int \frac{d^{3} k}{(2 \pi)^{3}} \int \frac{d^{3} p}{(2 \pi)^{3}} \operatorname{Tr}\left[\gamma_{0} G_{0}(-p+q) \gamma_{0} G_{0}(k+p+q) \gamma_{0}\right. \\
& \left.\times G_{0}(k+p) \gamma_{0} D(p) G_{0}(-p)\right]
\end{aligned}
$$

Perform a variable shift, $k \rightarrow k-p$, for the second term, then

$$
\Pi_{\mathrm{v}}(q)=2 N \operatorname{Tr}\left[X(q) \int \frac{d^{3} k}{(2 \pi)^{3}} G_{0}(k) \gamma_{0} G_{0}(k+q)\right],
$$

where

$$
X(q)=-\int \frac{d^{3} p}{(2 \pi)^{3}} \gamma_{0} G_{0}(p+q) \gamma_{0} G_{0}(p) \gamma_{0} D(p) .
$$

The most leading term is found to be

$$
X(q)=-\frac{\gamma_{0}}{4 \pi^{2}} \ln \left(\frac{\Lambda}{q}\right)\left(\frac{e^{2}}{2 \epsilon_{0} v_{F}}\right) \int_{0}^{\pi} d \theta \frac{\cos ^{2} \theta-\sin ^{2} \theta}{1+\frac{N e^{2}}{16 \epsilon_{0} v_{F}} \sin \theta} .
$$

In the large $N$ limit, it is possible to use the approximation

$$
\int_{0}^{\pi} d \theta \frac{\cos ^{2} \theta-\sin ^{2} \theta}{1+\frac{N e^{2}}{16 \epsilon_{0} v_{F}} \sin \theta} \approx \frac{32 \epsilon_{0} v_{F}}{N e^{2}} \ln \left(N \frac{e^{2}}{8 \epsilon_{0} v_{F}}\right),
$$


so that

$$
X(q)=-\frac{4 \gamma_{0}}{\pi^{2}} \frac{\ln \left(N \frac{e^{2}}{8 \epsilon_{0} v_{F}}\right) \ln \left(\frac{\Lambda}{q}\right)}{N} .
$$

After substituting Eq.(18) to Eq.(14), we finally get

$$
\Pi_{\mathrm{v}}(q)=-\frac{8}{\pi^{2}} \frac{\ln \left(N \frac{e^{2}}{8 \epsilon_{0} v_{F}}\right) \ln \left(\frac{\Lambda}{q}\right)}{N} \Pi(q) .
$$

[1] Castro Neto A H, Guinea F, Peres N M R, Novoselov K S and Geim A K, Rev. Mod. Phys. 81, 109 (2009).

[2] Das Sarma S, Adam S, Hwang E H and Rossi E, arXiv:1003.4731v2.

[3] Khveshchenko D V, Phys. Rev. Lett. 87, 246802 (2001); Khveshchenko D V and Leal H, Nucl. Phys. B 687, 323 (2004).

[4] Gorbar E V, Gusynin V P, Miransky V A and Shovkovy I A, Phys. Rev. B 66045108 (2002).

[5] Liu G-Z, Li W and Cheng G, Phys. Rev. B 79, 205429 (2009)

[6] Liu G-Z and Wang J-R, New J. Phys. 13, 033022 (2011).

[7] Gamayun O V, Gorbar E V and Gusynin V P, Phys. Rev. B 81, 075429 (2010).

[8] Hands S J and Strouthos C, Phys. Rev. B 78, 165423 (2008).

[9] Armour W, Hands S J, and Strouthos C, Phys. Rev. B 81, 125105 (2010).

[10] Drut J E and Lahde T A, Phys. Rev. Lett. 102, 026802 (2009).

[11] Drut J E and Lahde T A, Phys. Rev. B 79, 165425 (2009).

[12] Herbut I F, Phys. Rev. Lett. 97, 146401 (2006).

[13] Gusynin V P, Sharapov S G and Carbotte J P, Phys. Rev. Lett. 96, 256802 (2006).

[14] Kotov V N, Uchoa B and Castro Neto A H, Phys, Rev. B 80, 165424 (2009).

[15] Li W and G.-Z. Liu, Phys. Lett. A 374, 2957 (2010).

[16] Castro Neto A H, Physics 2, 30 (2009).

[17] Carbotte J P, Rev. Mod. Phys. 62, 1027 (1990).

[18] J. Polchinski, Nucl. Phys. B 422, 617 (1994).

[19] Wang J-R and Liu G-Z, Nucl. Phys. B 832, 441 (2010).

[20] Vojta M, Zhang y and Sachdev S, Phys. Rev. B 62, 6721 (2000).

[21] Khveshchenko D V and Paaske J, Phys. Rev. Lett. 86, 4672 (2001).

[22] Chubukov A V, Pepin C and Rech J, Phys. Rev. Lett. 92, 147003 (2004).

[23] Rech J, Pepin C and Chubukov A V, Phys. Rev. B 74, 195126 (2006).

[24] Dhokarh D and Chubukov A V, Phys. Rev. B 83, 064518 (2011).

[25] Aitchison I J R, Dorey N, Klein-Kreisler M and Mavromotos N E, Phys. Lett. B 294, 91 (1992).

[26] Dorey N and Mavromotos N E, Nucl. Phys. B 386, 614 (1992).

[27] Franz M, Pereg-Barnea T, Sheehy D E and Tesanovic Z, Phys. Rev. B 68, 024508 (2003). 